Title:

\title{
Corneal topography with an aberrometry-topography system
}

Authors:

Michael Mülhaupt, ${ }^{1}$ Sven Dietzko, ${ }^{1}$ James Wolffsohn, ${ }^{2}$ Stefan Bandlitz,,${ }^{1,2}$

${ }^{1}$ Höhere Fachschule für Augenoptik Köln (Cologne School of Optometry), Cologne, Germany

${ }^{2}$ Ophthalmic Research Group, Life and Health Sciences, Aston University, Birmingham, UK

Tables and Figures: Table and Figures

Word count: 2080

\section{Corresponding Author}

Stefan Bandlitz

Höhere Fachschule für Augenoptik Köln

(Cologne School of Optometry)

Bayenthalgürtel 6-8

D-50968 Köln, Germany

e-mail: bandlitz@hfak.de

Telephone: 0049-221-348080 


\section{ABSTRACT}

Purpose: To investigate the agreement between the central corneal radii and corneal eccentricity measurements generated by the new Wave Analyzer 700 Medica (WAV) compared to the Keratograph 4 (KER) and to test the repeatability of the instruments.

Methods: 20 subjects (10 male, mean age 29.1 years, range 21-50 years) were recruited from the students and staff of the Cologne School of Optometry. Central corneal radii for the flat $\left(r_{c / f l}\right)$ and steep $\left(r_{c / s t}\right)$ meridian as well as corneal eccentricity for the nasal (enas), temporal (etemp), inferior (einf) and superior (esup) directions were measured using WAV and KER by one examiner in a randomized order.

Results: Central radii of the flat (rc/fl) and steep (rc/st) meridian measured with both instruments were statically significantly correlated $(r=0.945$ and $r=0.951 ; p<0.001)$. Comparison between the WAV and KER showed that $r_{c / f l}$ and $r_{c / s t}$ measured with WAV were significantly steeper than those measured with KER $(p<0.001)$. Corneal eccentricities were statistically significantly correlated in all meridians $(p<0.05)$. Compared to KER, etemp and esup measured with WAV were greater $(p<0.05)$, while there were no statistically significant differences for $e_{\text {nas }}$ and $e_{\text {inf }}(p=0.350$ and $\mathrm{p}=0.083$ ). For the central radii, repeated measurements were not significantly different for the KER or WAV ( $p>0.05)$. Limits of agreement (LoA) indicate a better repeatability for the KER compared to WAV.

Conclusions: Corneal topography measurements captured with the WAV were strongly correlated with the KER. However, due to the differences in measured corneal 
radii and eccentricities, the devices cannot be used interchangeably. For corneal topography the KER demonstrated better repeatability.

Key words: Corneal topography, placido-based, corneal radius, corneal eccentricity, aberrometry-topography. 
1 The measurement of the shape, refractive power and thickness of the cornea is

2 essential for the planning of corneal refractive surgery, for diagnosis of corneal

3 diseases and for fitting contact lenses, in particular speciality lenses. Various

4 diagnostic procedures have been developed for the analysis of the corneal surface.

5 Corneal topographical measurements can be performed by classic Placido-based

6 topographers as well as by tomography systems that produce three-dimensional

7 corneal models from cross-sectional images [1].

8

9 Placido-based computerized videokeratoscopy, proposed first by Klyce in 1984 [2], are

10 the most frequently used corneal topography systems in clinical practice [3]. This

11 method of imaging of the anterior corneal surface analyses tear film reflected images

12 of multiple concentric rings projected on the cornea. In contrast, corneal tomography 13 provides an analysis of the shape of anterior and posterior corneal surfaces, as well

14 as the thickness distribution of the cornea [4]. Corneal tomography can be performed 15 by a scanned slit, rotating Scheimpflug cameras or by optical coherence tomography $16[5]$

Recently, a new corneal topography with an integrated aberrometry-topography system named the Wave Analyzer 700 Medica (Essilor, Freiburg, Germany) has been introduced to the market. The Wave Analyzer is a multifunctional device for performing

21 objective refraction, aberrometry, pupillometry, pachymetry, non-contact tonometry, measurement of anterior chamber depth and angle as well as corneal topography. The

23 instrument combines a Hartmann-Shack aberrometer, an air tonometer, a Scheimpflug 24 camera and a Placido-based topographer. However, the data for the corneal radii and 
corneal eccentricity is only generated from the Placido-disc measurement without any contribution of the Scheimpflug camera.

Consequently, the aims of this study were (i) to investigate the agreement in the measurement of central corneal radii and corneal eccentricity between the new Wave Analyzer 700 Medica (WAV) and the Placido-based Keratograph 4 (KER) (Oculus Optikgeräte GmbH, Wetzlar, Germany) and (ii) to test the repeatability of the instruments. 3

\section{MATERIALS AND METHODS}

\section{Instruments}

To measure central corneal radii as well as corneal eccentricity, two placido based corneal topographers were used in this study. The Keratograph 4 (Oculus Optikgeräte $\mathrm{GmbH}$, Wetzlar, Germany) uses a placido cone consisting of 22 red illuminated rings $(650 \mathrm{~nm})$ at $80 \mathrm{~mm}$ from the eye to generate 22000 measuring points. The Wave

Analyzer 700 Medica (Essilor, Freiburg, Germany) is a diagnostic device that performs

objective refraction, aberrometry, pupillometry, crystalline lens opacity, pachymetry,

3 tonometry and topography. For corneal topography it uses a placido cone off 24 rings

4 to generate 6144 measuring points. Instruments had been calibrated following the 

contact lenses during the preceding 24 hours prior to the study.

\section{In Vitro Study}

\section{In Vivo Study}

Four precision glass balls (radii: 6.00, 7.00, 8.00 and $9.00 \mathrm{~mm}$; CA 100-Caldev, Topcon, Tokyo, Japan) were used as a model of the cornea. The mean of three consecutive measurements of the four glass balls was compared between the KER and the WAV at two different sessions at the same time of day (day 1 and day 2).

Twenty healthy subjects (mean age $29.1 \pm 9.2$ (SD) years, range 21 to 50 years, even male to female split) were recruited from the students and staff of the Höhere Fachschule für Augenoptik Köln (Cologne School of Optometry), Cologne, Germany. All subjects underwent a medical history and a slit lamp examination. Subjects were excluded if: they had a current or previous condition known to affect the cornea, conjunctiva or the sclera such as pterygium and pinguecula; had a history of previous ocular surgery, including refractive or strabismus surgery, eyelid surgery, or corneal surgery; had any previous ocular trauma; were diabetic; were taking medication known to affect the ocular surface or sclera; and/or had worn rigid contact lenses or soft 
The study was approved by the Research Ethics Committee and all subjects gave

69 written informed consent before participating in the study. The procedures were conducted in accordance with the requirements of the Declaration of Helsinki (1983)

71 and patient data were used only in anonymized form.

72

73 Central corneal radii for the flat $\left(r_{\mathrm{c} / \mathrm{fl}}\right)$ and steep $\left(\mathrm{r}_{\mathrm{c} / \mathrm{st}}\right)$ meridian as well as corneal 74 eccentricity for the nasal ( $\left.e_{n a s}\right)$, temporal ( $\left.e_{t e m p}\right)$, inferior ( $\left.e_{\text {inf }}\right)$ and superior ( $\left.e_{\text {sup }}\right)$ 75 direction were measured by one examiner using the WAV and the KER in a randomized order. Corneal eccentricities were taken from the data given for an angle of $30^{\circ}$. The mean of three consecutive measurements of the right eye was recorded for both instruments at two different sessions at the same time of day (day 1 and day 2).

\section{Statistical Analyses}

83 Normal distribution of data was analyzed by Shapiro-Wilk test. As the data was normally distributed, differences between sessions (day 1 and day 2) and instruments were analyzed using Bland-Altman plots, coefficient of repeatability (CR), and paired t-tests. The relationship between the WAV and KER measurements was analyzed by

88 Software Inc., Chicago, USA).

89

RESULTS

\section{In Vitro Study}


92 The measured radii of the four glass balls were $6.01,6.97,7.99$, and $8.99 \mathrm{~mm}$ for the

93 WAV and $6.02,7.01,8.00$, and $9.00 \mathrm{~mm}$ for the KER. The mean difference between

94 the measurements of the two devices was $0.018 \mathrm{~mm}(95 \%$ confidence interval [Cl], -

950.015 to $+0.050 \mathrm{~mm} ; \mathrm{p}=0.125$ ) (Figure 5). Repeated measurements from day 1 and day 2 were not significantly different for the KER (paired t-test: $p=0.391$ ), but they were different for the WAV $(p=0.034)$. The mean difference and the limits of agreement (LoA) indicate a better in vitro repeatability for the KER $(0.005 \mathrm{~mm}$; LoA 0.013 to $0.008 \mathrm{~mm})$ compared to the WAV $(0.030 \mathrm{~mm}$; LoA -0.003 to $+0.118 \mathrm{~mm})$.

\section{In Vivo Study}

Table 1 summarizes the mean values \pm standard deviations of central corneal radii and corneal eccentricities, mean difference and limits of agreement (LoA) of the two measuring sessions (day 1 to day 2 ) and the mean differences and $95 \%$ confidence interval between the two instruments.

Central corneal radii of the flat $\left(\mathrm{r}_{\mathrm{c} / \mathrm{fl}}\right)$ and steep $\left(\mathrm{r}_{\mathrm{c} / \mathrm{st}}\right)$ meridian measured with both instruments were statically significantly correlated $(r=0.945$ and $r=0.951$; both $\mathrm{p}<0.001)$. On average the mean central radii measured with the WAV were significantly steeper than those measured with the KER $(-0.05 \mathrm{~mm}$; Cl -0.08 to -0.02 ; paired t-test; $\mathrm{p}<0.001$ ) (Figure 6).

The measured corneal eccentricities were statistically significantly correlated in all meridians (enas; $;=0.747$, etemp; $r=0.541$, e $e_{\text {inf }} ; r=0.783$ and superior $e_{\text {sup }} ; r=0.661$; all $\mathrm{p}<0.05)$. On average the mean corneal eccentricities measured with the WAV were significantly greater than those measured with the KER $(+0.06$; $\mathrm{Cl} 0.0126$ to 0.105 ; paired t-test; $p=0.009$ ) (Figure 7). Compared to the KER, etemp and $e_{\text {sup }}$ measured with 
118 the WAV were greater $(p<0.05)$, while there were no statistically significant differences

119 for enas and $\operatorname{einf}(p=0.350$ and $p=0.083)$ (Table 1).

121 For the central radii, repeated measurements from day 1 to day 2 were not significantly

122 different for the KER and WAV (paired t-test; $r c / f l: p=0.523$ and $p=0.860 ; r c / s t: p=0.783$

123 and $p=0.154)$. The mean difference and the limits of agreement (LoA) indicate a better

124 repeatability for the KER compared to the WAV (Table 1).

125

126 For the overall corneal eccentricity, repeated measurements from day 1 to day 2 were 127 not significantly different for the KER and the WAV (paired t-test; $p>0.05$ ). The mean 128 difference and the limits of agreement (LoA) indicate a better repeatability for the KER 129 compared to the WAV (Table 1).

\section{DISCUSSION}

133 The Wave Analyzer is a multifunctional device for performing objective refraction, 134 aberrometry, pupillometry, pachymetry, non-contact tonometry and corneal 135 topography. Comparing the values obtained for corneal topography with those of a 136 placido-based Keratograph 4 showed a high correlation. However, radii measured with 137 the Wave Analyzer were, on average, $0.06 \mathrm{~mm}$ and $0.09 \mathrm{~mm}$ (flat or steep meridian) 138 steeper than those of the Keratograph 4.

140 Shneor et al. [6] compared the L80 (Visionix Luneau, Chartes, France), a multi-function 141 device similar to the Wave Analyzer, with a manual Bausch \& Lomb ophthalmometer. 142 As in the present study, they report statistically significantly steeper central radii 143 measurements (by $0.05 \mathrm{~mm}$ and $0.07 \mathrm{~mm}$ in the flat or steep meridians respectively) 
144 compared to the manual ophthalmometer. For the Keratograph 4 (Oculus, Germany),

145 Best et al. reported flatter central corneal radii compared to Tonoref II (Nidek, Japan) 146 [7]

148 Likewise, a comparison of the Placido-based Allegro Topolyzer system (Alcon 149 Research, Ltd., Fort Worth, TX, USA) with a Scheimpflug camera-based Galilei G4 150 system (Ziemer Ophthalmic Systems AG, Port, Switzerland) showed statistically 151 significant differences in the central corneal radii [8]. The Scheimpflug camera-based system showed steeper radii than the Placido-based system; the differences in 153 patients with keratoconus were even greater [8,9]. Comparing the Orbscan II (Orbtek), 154 a combination of a slit scanning technique and Placido disc image, with the Palcido155 based EyeSys (Houston, TX, USA), Douthwaite and Mallen [10] found that the 156 Orbscan appears to under-read slightly for both apical radius and p-value.

158 In contrast, Laursen et al. [11] reported no significant differences in the measurement 159 of mean corneal power between different devices: Keratograph 4, Pentacam (Oculus, 160 Germany), Tonoref II (Nidek, Japan), IOLMaster 500 and Lensstar LS 900 (Haag-Streit, 161 Switzerland). A comparison of three Scheimpflug camera-based systems (Pentacam, 162 Galilei G2 and Sirus 3D) in a study by Hernández-Camarena et al. [12] also did not 163 show any statistically significant differences in the measurement of the central corneal 164 radii.

166 For corneal eccentricities, significant differences (mean differences from 0.08 to 0.26 ) 167 were found comparing four topographers (Humphrey, Atlas 991 (Zeiss), Dicon CT200 168 (Dicon, US), Orbscan II (Orbtek) and Medmont E300 (Medmont, Australia)) [13], which 
169 is in concordance to the mean differences of 0.07 and 0.08 reported for the temporal and superior eccentricities in the present study.

172 Furthermore, in the present study, a better in vivo repeatability of the measurements 173 was obtained for the Keratograph 4 compared to the WaveAnalyzer. The values for 174 the Keratograph 4 described in this study are in good agreement with repeatability described by Riede-Pult et al. [14] for the Keratograph 2. Device-specific differences in the repeatability of the measurement of central corneal radii as well as corneal eccentricities have already been reported in several studies [11-13, 15, 16].

For the differences in measurement and in repeatability described in the various 180 studies, several causes can be considered: differences in the measuring principle 181 (manual keratometry, Placido-based systems, Scheimpflug camera-based systems); 182 differences in the measured area of the cornea (e.g. number of Placido-rings); different 183 calculation algorithms of the devices; as well as differences between the subjects (eg. 184 keratokonus or dry eye). Hamer et al. suggested, that the Placido-based systems seem 185 to be more susceptible to changes in the tear film than the Scheimpflug camera-based systems [16]. Corneal topographers such as those utilising a Placido disc, analyse the pattern of light rays reflected off the cornea and tear film-air interface and therefore any disruption of the tear film may influence the measurement [16]. Since the reflection 189 quality of the placido mires indicates the quality of the tear film over time, topographers 190 can also be used to assess tear film stability [7].

192 A limitation of the present study results from the eye models used for the in vitro study. 193 The glass balls had spherical surfaces which does not ideally reflects the aspherical 194 shape of most corneas. Therefore, Douthwaite [17] proposed the use of conicoidal 
195

Practitioners should be aware of the measuring accuracy and the repeatability of the

214 topography instrument used. This is important for the appropriate selection of the first 215 contact lens to be trialled, as well as for the diagnosis and monitoring of corneal 216 changes, especially when different topography systems are in use.

\section{CONCLUSIONS}


220 Comparing the corneal topography determined by the Wave Analyzer with that of the

221 Keratograph 4 showed a high correlation. However, due to the differences in measured 222 corneal radii and eccentricities, the devices cannot be used interchangeably. For 223 corneal topography the KER demonstrated better repeatability.

\section{Conflict of interest}

227 None

\section{REFERENCES}

241 [1] Herrmann C, Ludwig U, Duncker G. [Corneal topography. Analysis of the corneal 242 surface]. Ophthalmologe. 2008;105:193-204; quiz 5-6.

243 [2] Klyce SD. Computer-assisted corneal topography. High-resolution graphic 244 presentation and analysis of keratoscopy. Invest Ophthalmol Vis Sci. 1984;25:142635. 
246 [3] Fung MW, Raja D, Fedor P, Kaufman SC. Corneal Topography and Imaging 247 emedicinemedscapecom/article/1196836 2016.

248 [4] Gokul A, Vellara HR, Patel DV. Advanced anterior segment imaging in keratoconus:

249 a review. Clinical \& experimental ophthalmology. 2017.

250 [5] Fan R, Chan TC, Prakash G, Jhanji V. Applications of corneal topography and

251 tomography: a review. Clinical \& experimental ophthalmology. 2017.

252 [6] Shneor E, Millodot M, Zyroff M, Gordon-Shaag A. Validation of keratometric 253 measurements obtained with a new integrated aberrometry-topography system. 254 Journal of Optometry. 2012;5:80-6.

255 [7] Best N, Drury L, Wolffsohn JS. Clinical evaluation of the Oculus Keratograph. Cont 256 Lens Anterior Eye. 2012;35:171-4.

257 [8] Ortiz-Toquero S, Zuniga V, Rodriguez G, de Juan V, Martin R. Agreement of corneal 258 measurements between dual rotating Scheimpflug-Placido system and Placido-based 259 topography device in normal and keratoconus eyes. J Cataract Refract Surg. $260 \quad 2016 ; 42: 1198-206$.

261 [9] Stefano VS, Melo Junior LA, Mallmann F, Schor P. Interchangeability between 262 Placido disc and Scheimpflug system: quantitative and qualitative analysis. Arquivos brasileiros de oftalmologia. 2010;73:363-6.

264 [10] Douthwaite WA, Mallen EA. Cornea measurement comparison with Orbscan II 265 and EyeSys videokeratoscope. Optom Vis Sci. 2007;84:598-604.

266 [11] Laursen JV, Jeppesen P, Olsen T. Precision of 5 different keratometry devices. 267 Int Ophthalmol. 2016;36:17-20.

268 [12] Hernandez-Camarena JC, Chirinos-Saldana P, Navas A, Ramirez-Miranda A, de 269 la Mota A, Jimenez-Corona A, et al. Repeatability, reproducibility, and agreement 270 between three different Scheimpflug systems in measuring corneal and anterior 271 segment biometry. J Refract Surg. 2014;30:616-21. 
272 [13] Cho P, Lam AK, Mountford J, Ng L. The performance of four different corneal

273 topographers on normal human corneas and its impact on orthokeratology lens fitting.

274 Optom Vis Sci. 2002;79:175-83.

275 [14] Riede-Pult BH, Evans K, Pult H. Investigating the Short-term Effect of Eyelid 276 Massage on Corneal Topography. Optom Vis Sci. 2017;94:700-6.

277 [15] Mao X, Savini G, Zhuo Z, Feng Y, Zhang J, Wang Q, et al. Repeatability, 278 reproducibility, and agreement of corneal power measurements obtained with a new 279 corneal topographer. J Cataract Refract Surg. 2013;39:1561-9.

280 [16] Hamer CA, Buckhurst H, Purslow C, Shum GL, Habib NE, Buckhurst PJ. 281 Comparison of reliability and repeatability of corneal curvature assessment with six 282 keratometers. Clin Exp Optom. 2016;99:583-9.

283 [17] Douthwaite WA. EyeSys corneal topography measurement applied to calibrated 284 ellipsoidal convex surfaces. Br J Ophthalmol. 1995;79:797-801.

285 [18] Lorian V. In vitro simulation of in vivo conditions: physical state of the culture 286 medium. Journal of clinical microbiology. 1989;27:2403-6.

287 [19] Atchison DA, Thibos LN. Optical models of the human eye. Clin Exp Optom. $288 \quad 2016 ; 99: 99-106$.

289 [20] McMahon TT, Anderson RJ, Joslin CE, Rosas GA, Collaborative Longitudinal 290 Evaluation of Keratoconus Study Topography Analysis G. Precision of three 291 topography instruments in keratoconus subjects. Optom Vis Sci. 2001;78:599-604.

292 [21] McMahon TT, Anderson RJ, Roberts C, Mahmoud AM, Szczotka-Flynn LB, 293 Raasch TW, et al. Repeatability of corneal topography measurement in keratoconus 294 with the TMS-1. Optom Vis Sci. 2005;82:405-15. 
318 Figures

320 Figure 1. Wave Analyzer 700 Medica (Essilor, Freiburg, Germany).

322 Figure 2. Keratograph 4 (Oculus GmbH, Wetzlar, Germany). 
324 Figure 3. Output of the Wave Analyzer 700 (Essilor, Freiburg, Germany).

326 Figure 4. Output of the Keratograph 4 (Oculus $\mathrm{GmbH}$, Wetzlar, Germany).

328 Figure 5. In vitro difference in mean radius $(\mathrm{mm})$ between the Keratograph 4 and the 329 Wave Analyzer.

331 Figure 6. In vivo difference in mean radius $(\mathrm{mm})$ between the Keratograph 4 and the Wave Analyzer (solid line: mean; dashed line: $95 \%$ confidence interval).

334 Figure 7. In vivo difference in mean eccentricity between the Keratograph 4 and the 335 Wave Analyzer (solid line: mean; dashed line: 95\% confidence interval).

Tables

339 Table 1. Mean values \pm standard deviations of three repeated measurements of 340 central corneal radii and corneal eccentricities, mean difference and limits of 341 agreement (LoA) of two measuring sessions (day 1 to day 2 ) and the mean differences 342 and $95 \%$ confidence interval between both instruments ( $n=20$ eyes). *Indicates 343 statistically significant differences. 
Table 1

\begin{tabular}{|c|c|c|c|c|c|c|c|c|}
\hline & $\begin{array}{c}\text { Wave } \\
\text { Analyzer }\end{array}$ & $\begin{array}{c}\text { Mean Difference } \\
(95 \% \text { LoA) } \\
\text { Day1 to Day } 2 \\
\end{array}$ & $p$ value & Keratograph & $\begin{array}{c}\text { Mean Difference } \\
(95 \% \text { LoA) } \\
\text { Day } 1 \text { to Day } 2\end{array}$ & $p$ value & $\begin{array}{c}\text { Mean Difference } \\
(95 \% \mathrm{Cl}) \\
\text { KER - WAV }\end{array}$ & $p$ value \\
\hline \multicolumn{9}{|l|}{ Central corneal radii } \\
\hline Flat meridian $\left(\mathrm{r}_{\mathrm{c} / \mathrm{fl}}\right)$ & $7.82 \pm 0.26$ & $-0.01(-0.26$ to 0.25$)$ & $p=0.860$ & $7.88 \pm 0.27$ & $+0.01(-0.08$ to 0.09$)$ & $p=0.594$ & $-0.06(-0.10$ to -0.02$)$ & $p=0.006^{\star}$ \\
\hline Steep meridian $\left(r_{c / s t}\right)$ & $7.62 \pm 0.30$ & $+0.02(-0.15$ to 0.20$)$ & $p=0.308$ & $7.71 \pm 0.26$ & $0.00(-0.06$ to 0.06$)$ & $p=0.783$ & $-0.09(-0.17$ to -0.01$)$ & $\mathrm{p}<0.001$ \\
\hline \multicolumn{9}{|l|}{ Corneal eccentricity } \\
\hline Nasal (enas) & $0.71 \pm 0.24$ & $+0.01(-0.36$ to 0.38$)$ & $p=0.810$ & $0.68 \pm 0.11$ & $-0.02(-0.11$ to 0.14$)$ & $p=0.469$ & $+0.04(-0.04$ to +0.12$)$ & $p=0.350$ \\
\hline Temporal (etemp) & $0.50 \pm 0.39$ & $+0.01(-0.78$ to 0.79$)$ & $p=0.340$ & $0.43 \pm 0.08$ & $-0.01(-0.12$ to 0.11$)$ & $p=0.615$ & $+0.07(-0.10$ to +0.23$)$ & $p=0.014^{\star}$ \\
\hline Inferior (e & $0.56 \pm 0.19$ & $-0.02(-0.29$ to 0.25$)$ & $\mathrm{p}=0.496$ & $0.51 \pm 0.15$ & $0.00(-0.12$ to 0.11$)$ & $p=0.823$ & $+0.05(-0.01$ to +0.11$)$ & $p=0.083$ \\
\hline Superior (esup) & $0.61 \pm 0.14$ & $+0.03(-0.77$ to 0.82$)$ & $p=0.090$ & $0.53 \pm 0.13$ & $+0.01(-0.18$ to 0.21$)$ & $p=0.402$ & $+0.08(+0.03$ to +0.13$)$ & $p=0.004^{*}$ \\
\hline Overall & $0.60 \pm 0.26$ & $+0.04(-0.50$ to 0.49$)$ & $p=0.592$ & $0.53 \pm 0.15$ & $0.00(-0.13$ to 0.12$)$ & $\mathrm{p}=0.780$ & $+0.06(+0.01$ to +0.11$)$ & $p=0.009^{*}$ \\
\hline
\end{tabular}


Figure 1

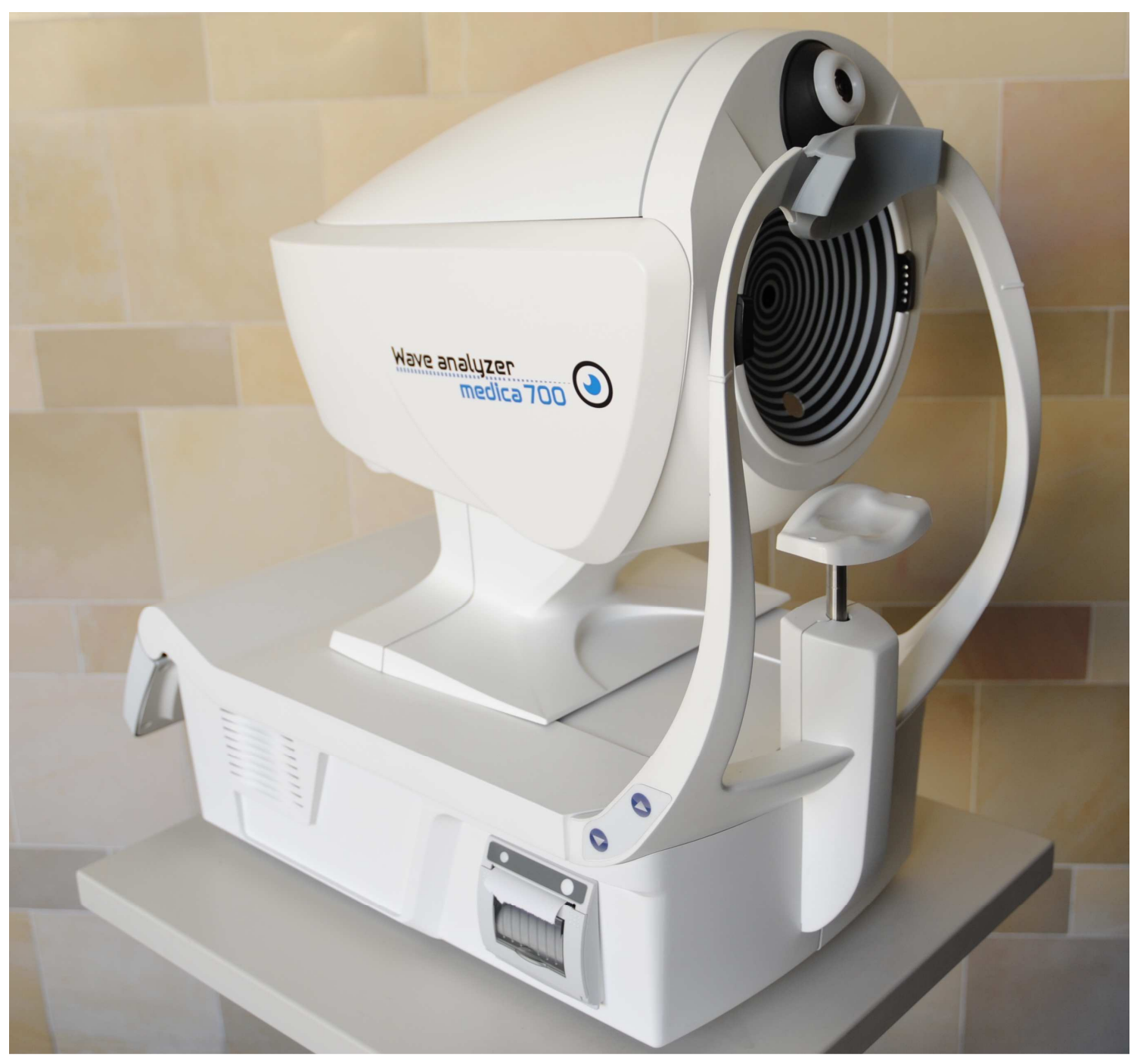


Figure 2

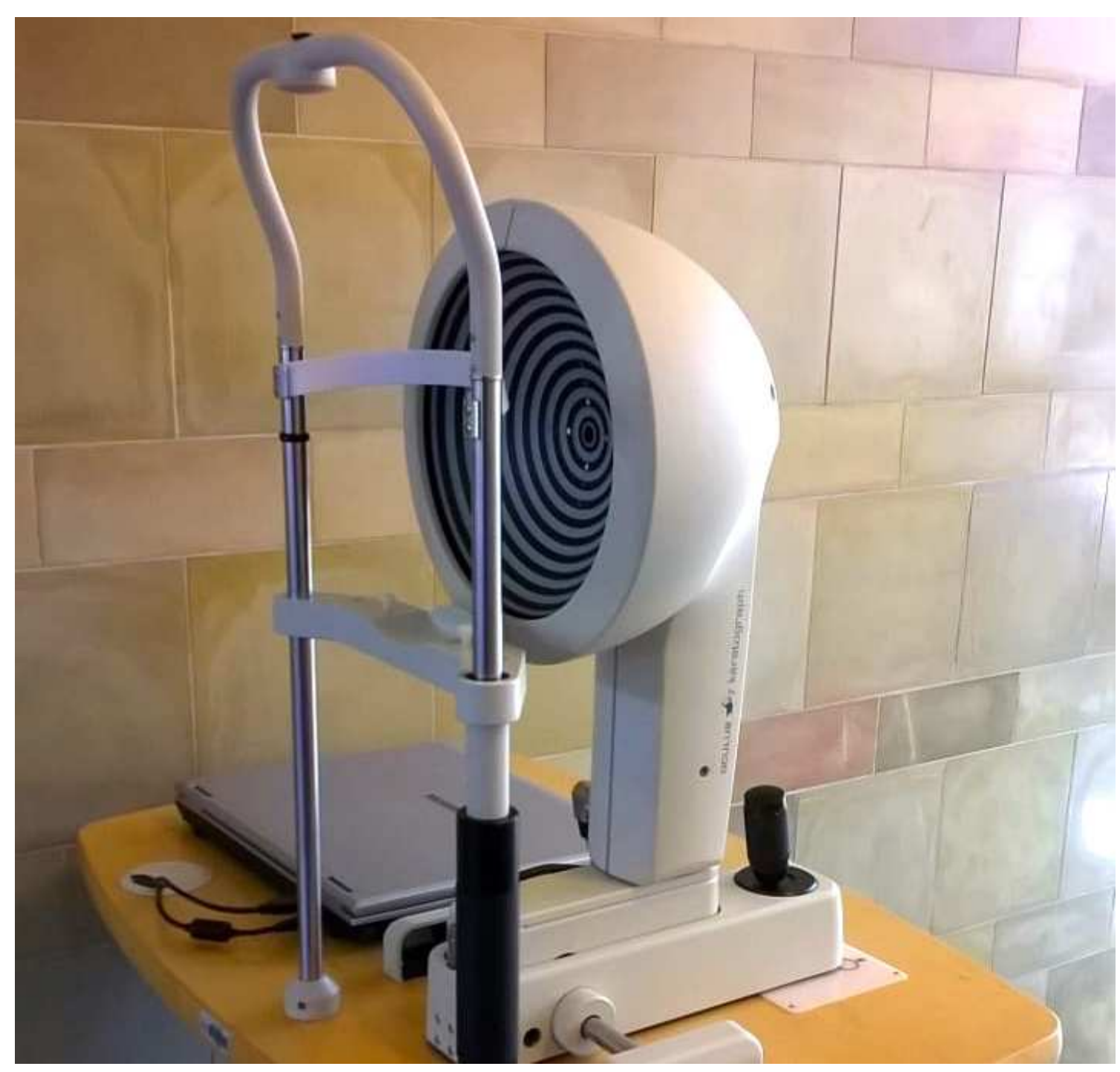


Figure 3

\section{(1) essilor}

\section{$\mathrm{R}$ ()}

เक

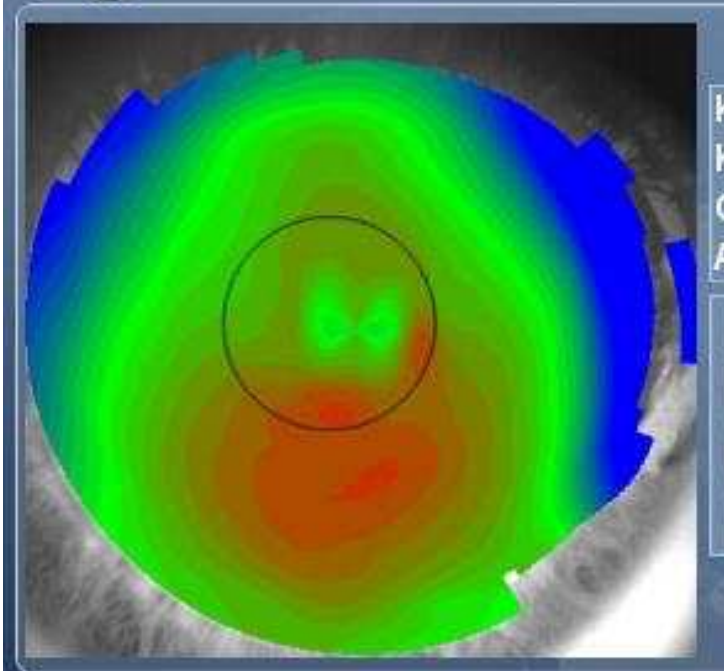

\section{Topography}

K1: 8.34mm@3 ${ }^{\circ}$ K1:

K2: $8.22 \mathrm{~mm} @ 93^{\circ} \quad \mathrm{K} 2$ :

Cyl: $-0.50 D @ 3^{\circ} \quad$ Cyl:

AVC: $8.28 \mathrm{~mm}$ AVG:

KPI: $\quad 0 \%$

HOA (rm): 0.29

LOA(pm): 0.45

Z(4.0):0.22@70

\begin{tabular}{|c|c|c|c|c|c|c|}
\hline \multicolumn{3}{|c|}{$3 \mathrm{~mm}$} & \multicolumn{2}{c|}{$5 \mathrm{~mm}$} & \multicolumn{2}{c|}{$7 \mathrm{~mm}$} \\
\hline K1 & $8.35 \mathrm{~mm}$ & $177^{\circ}$ & $8.36 \mathrm{~mm}$ & $4^{\circ}$ & $8.43 \mathrm{~mm}$ & $3^{\circ}$ \\
\hline K2 & $8.25 \mathrm{~mm}$ & $87^{\circ}$ & $8.25 \mathrm{~mm}$ & $94^{\circ}$ & $8.27 \mathrm{~mm}$ & $9^{\circ}$ \\
\hline AVG & $8.30 \mathrm{~mm}$ & & $8.31 \mathrm{~mm}$ & & $8.35 \mathrm{~mm}$ & \\
\hline CY1 & $-0.50 \mathrm{D}$ & $177^{\circ}$ & $-0.50 \mathrm{D}$ & $4^{\circ}$ & $-0.75 \mathrm{D}$ & $3^{\circ}$ \\
\hline
\end{tabular}

\begin{tabular}{|c|c|c|c|}
\hline & $3 \mathrm{~mm}$ & $5 \mathrm{~mm}$ & $7 \mathrm{~mm}$ \\
\hline $\mathrm{K} 1$ & & & \\
\hline $\mathrm{k} 2$ & & & \\
\hline AVG & & & \\
\hline cyl & & & \\
\hline
\end{tabular}


Figure 4
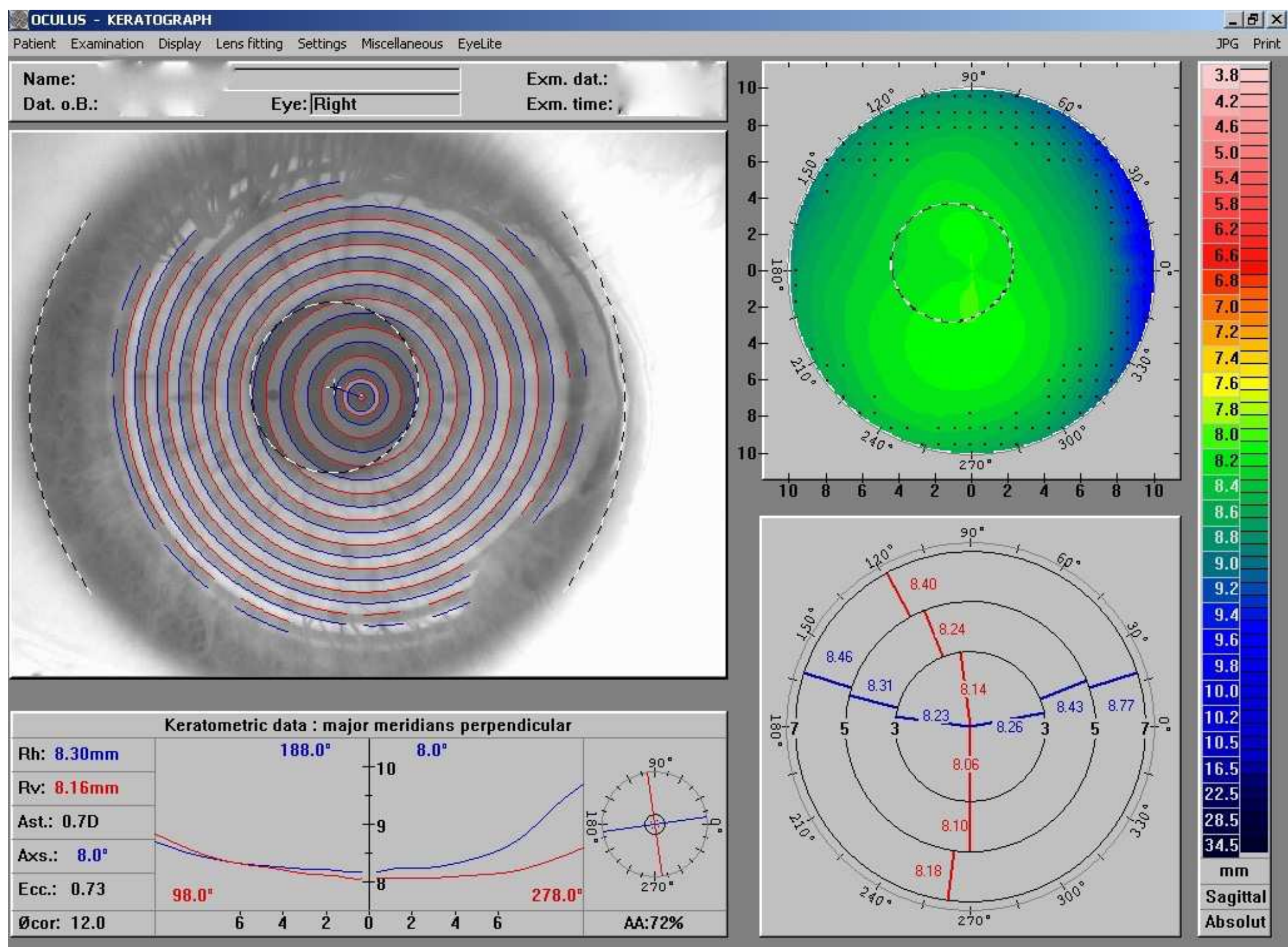


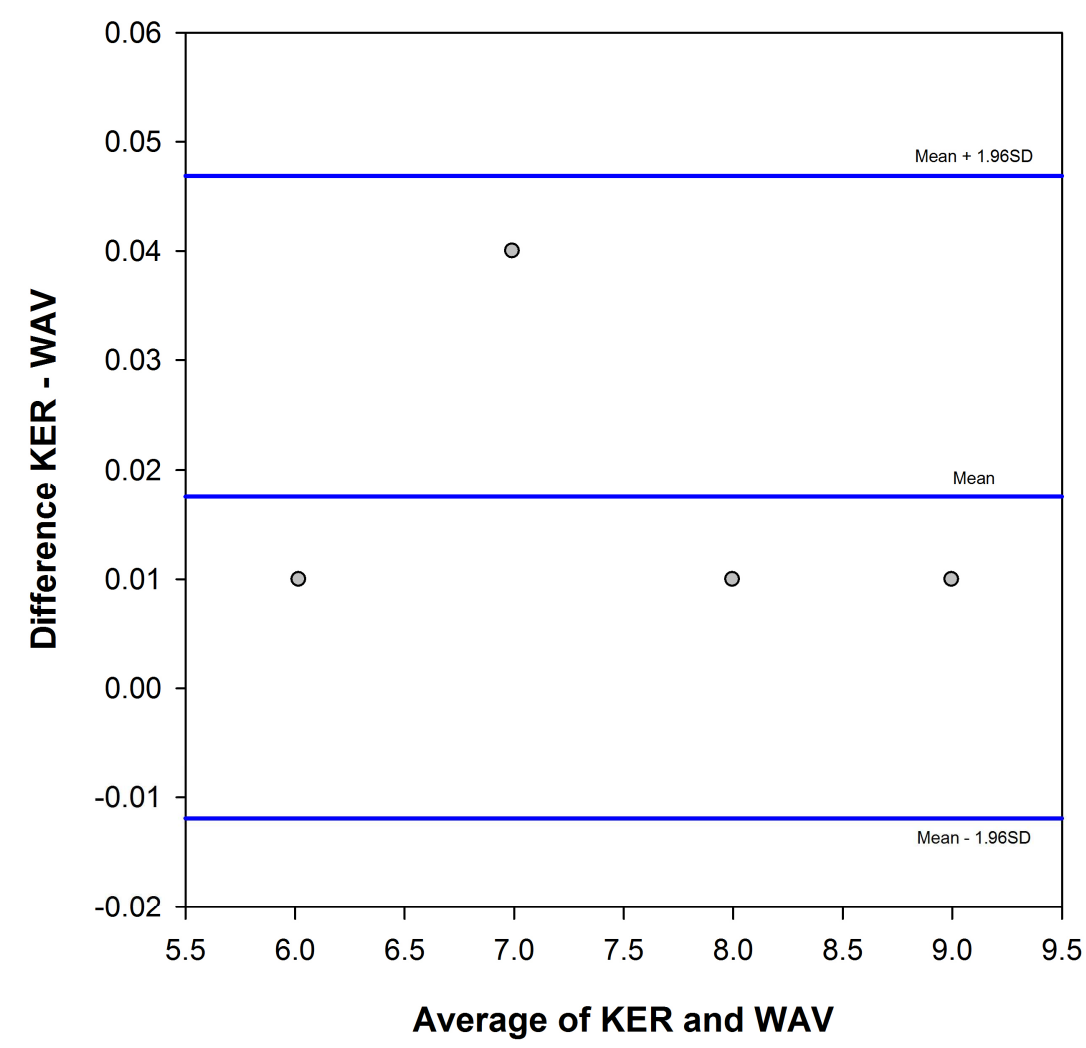


Figure 6

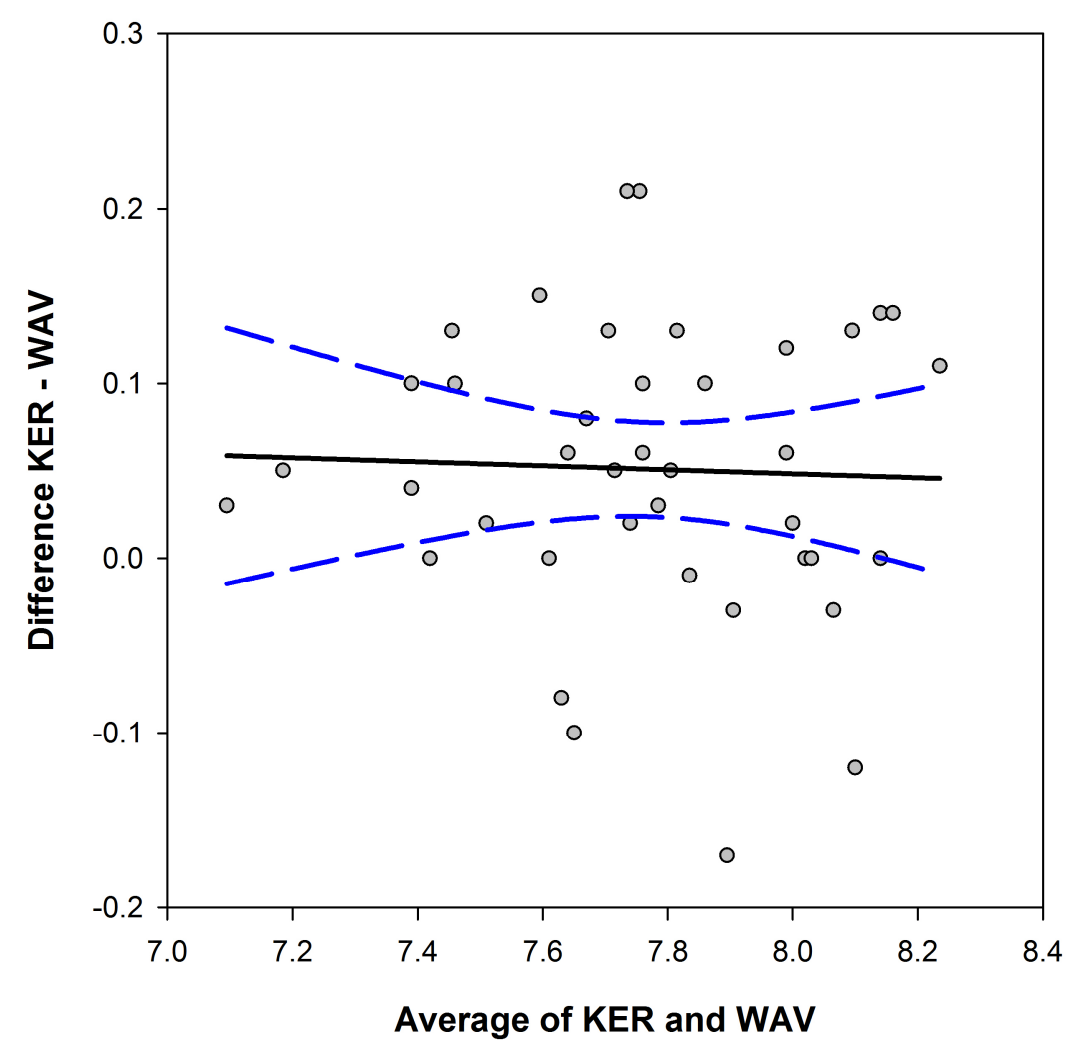


Figure 7

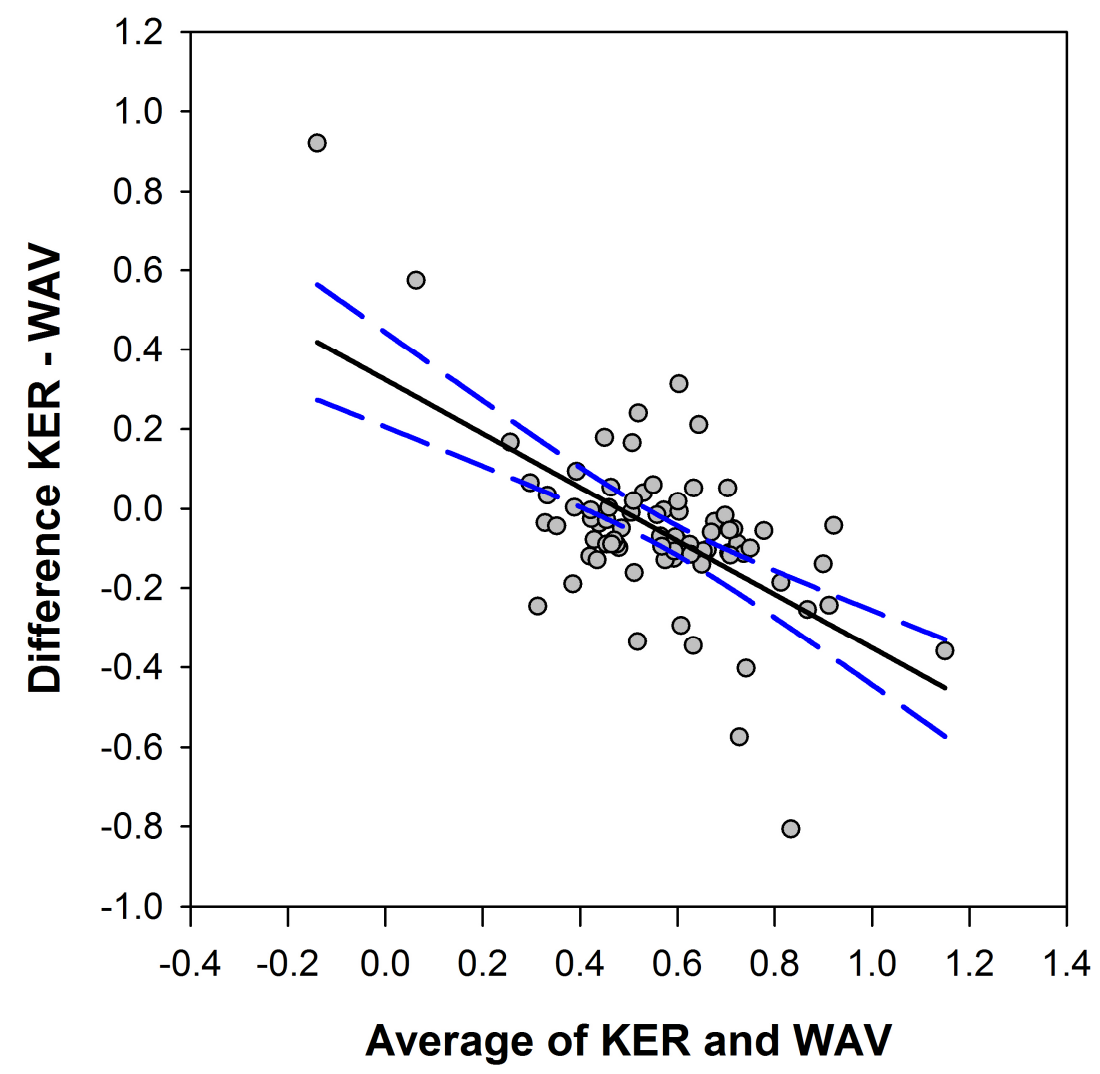

